\title{
Nasopharyngeal Pneumococcal Density and Evolution of Acute Respiratory Illnesses in Young Children, Peru, 2009-2011
}

\author{
Roger R. Fan, Leigh M. Howard, \\ Marie R. Griffin, Kathryn M. Edwards, \\ Yuwei Zhu, John V. Williams, Jorge E. Vidal, \\ Keith P. Klugman, Ana I. Gil, \\ Claudio F. Lanata, Carlos G. Grijalva
}

We examined nasopharyngeal pneumococcal colonization density patterns surrounding acute respiratory illnesses (ARI) in young children in Peru. Pneumococcal densities were dynamic, gradually increasing leading up to an ARI, peaking during the ARI, and decreasing after the ARI. Rhinovirus coinfection was associated with higher pneumococcal densities.

Streptococcus pneumoniae commonly colonizes the $\mathcal{N}$ nasopharynx of young children (1). Nasopharyngeal colonization density is relevant for transmission of bacteria and pathogenesis of pneumococcal diseases (2). Few studies have evaluated the longitudinal relationship between nasopharyngeal pneumococcal density and acute respiratory illnesses (ARIs). We examined the evolution of nasopharyngeal pneumococcal density surrounding ARIs in young children.

\section{The Study}

We performed sequential cross-sectional assessments from a prospective cohort study of Andean children in Peru (3). During 2009-2011, children $<3$ years of age from the District of San Marcos, Cajamarca, Peru, were assessed for ARIs during weekly household visits. The population was rural and had low incomes and limited access to healthcare $(3,4)$. Use of 7-valent pneumococcal conjugate vaccine (PCV7) started in late 2009. Institutional review boards of Vanderbilt University (Nashville, TN, USA) and the Instituto de Investigacion Nutricional (Lima, Peru) approved the study.

An ARI episode was defined as the length of time a child had cough or fever $(5,0)$. If a child was ill during a household visit, we assessed for pneumonia or lower

Author affiliations: Vanderbilt University, Nashville, Tennessee, USA (R.R. Fan, L.M. Howard, M.R. Griffin, K.M. Edwards, Y. Zhu, C.F. Lanata, C.G. Grijalva); University of Pittsburgh, Pittsburgh, Pennsylvania, USA (J.V. Williams); Emory University, Atlanta, Georgia, USA (J.E. Vidal, K.P. Klugman); Instituto de Investigacion Nutricional, Lima, Peru (A.I. Gil, C.F. Lanata)

DOI: http://dx.doi.org/10.3201/eid2211.160902 respiratory tract infection using IMCI-WHO (Integrated Management of Childhood Illness-World Health Organization) criteria $(5,7)$. If the child had an ARI during the preceding 7 days, we collected a nasal swab sample and tested it for respiratory viruses by reverse transcription PCR at Vanderbilt University $(6,8-11)$. Nasopharyngeal swab samples were collected monthly without regard to ARI and tested at Emory University (Atlanta, GA, USA) by using quantitative PCR for pneumococcal density determinations. For this study, we used samples collected in 2009 and 2011, representing periods before and after routine PCV7 use (12) (online Technical Appendix, http://wwwnc.cdc. gov/EID/article/22/11/16-0902-Techapp1.pdf).

Nasopharyngeal samples were classified according to their collection time surrounding ARIs: peri-ARI periods included pre-ARI (8-14 or 1-7 days before an ARI) and post-ARI (1-7 or 8-14 days after an ARI). Samples outside these periods were considered non-ARI samples. We compared log-transformed pneumococcal nasopharyngeal densities of samples from ARI, peri-ARI, and non-ARI periods by using multivariable quantile regression with robust SEs and adjusting for relevant covariates.

In secondary analyses, we assessed the role of respiratory viruses on pneumococcal density in children with ARIs. Because detection of nonrhinovirus respiratory viruses in nasal swabs was infrequent, we grouped samples into 4 distinct groups: rhinovirus only, rhinovirus and other viruses, other viruses only, and negative for any viruses.

We examined the role of pneumococcal acquisition on pneumococcal density using pneumococci-positive nasopharyngeal samples from children who had a sample collected within the preceding 60 days. Samples were categorized as 1) new colonization if the prior sample was negative, 2) serotype persistence if the prior sample was the same serotype, and 3) serotype replacement if the prior sample was a different serotype. If either serotype was nontypeable or unknown, the pattern was considered undetermined.

We assessed 3,579 nasopharyngeal samples from 833 children: 450 (12.6\%) were collected during ARIs, 956 (26.7\%) during peri-ARI periods, and 2,173 (57.8\%) during non-ARI periods. The median age was 1.39 years. The median duration for ARIs was 8 days (interquartile range [IQR] 5-13 days). According to IMCI-WHO criteria, 33 samples were associated with pneumonia or severe pneumonia (13) (Table). 
Pneumococcal Density and Acute Respiratory IIIness

Table. Demographic characteristics for children from whom nasopharyngeal swab samples were collected during different periods surrounding ARIs, Peru, 2009-2011*

\begin{tabular}{|c|c|c|c|c|c|c|c|}
\hline \multirow[b]{3}{*}{ Characteristic } & \multicolumn{6}{|c|}{ Period of sample collection } & \multirow[b]{3}{*}{$\begin{array}{c}\text { Total, } \\
\mathrm{N}=3,579\end{array}$} \\
\hline & \multirow[b]{2}{*}{$\begin{array}{l}\text { Non-ARI, } \\
n=2,173\end{array}$} & \multicolumn{2}{|c|}{ Pre-ARI } & \multirow[b]{2}{*}{$\begin{array}{c}\text { Current ARI, } \\
n=450\end{array}$} & \multicolumn{2}{|c|}{ Post-ARI } & \\
\hline & & $\begin{array}{c}8-14 \text { days } \\
n=211\end{array}$ & $\begin{array}{c}1-7 \text { days, } \\
n=222\end{array}$ & & $\begin{array}{c}1-7 \text { days } \\
n=332\end{array}$ & $\begin{array}{c}8-14 \text { days } \\
n=191\end{array}$ & \\
\hline No. childrent & 765 & 186 & 189 & 320 & 262 & 172 & 833 \\
\hline \multicolumn{8}{|l|}{ Demographics } \\
\hline Median age, y, at sample collection & 1.45 & 1.36 & 1.37 & 1.23 & 1.25 & 1.37 & 1.39 \\
\hline Male & 50.5 & 56.4 & 52.3 & 52.9 & 49.4 & 48.2 & 51.1 \\
\hline Attend daycare equivalent & 7.8 & 7.1 & 8.6 & 4.2 & 6.6 & 5.2 & 7.1 \\
\hline \multicolumn{8}{|l|}{ Patient's home } \\
\hline Traditional stoves for cooking & 63.1 & 73.5 & 63.5 & 61.1 & 68.7 & 62.8 & 64.0 \\
\hline Running water & 24.7 & 17.5 & 17.6 & 24.0 & 18.7 & 27.2 & 23.3 \\
\hline Sewer or septic tank & 21.1 & 18.5 & 18.5 & 20.4 & 22.3 & 22.5 & 20.9 \\
\hline Electricity & 42.3 & 34.6 & 37.4 & 38.7 & 40.7 & 41.9 & 40.9 \\
\hline \multicolumn{8}{|l|}{ Season and year at sample collection } \\
\hline Fall 2009 & 4.4 & 8.1 & 6.8 & 9.8 & 6.0 & 6.8 & 5.7 \\
\hline Winter 2009 & 19.3 & 25.1 & 27.5 & 30.0 & 23.2 & 25.7 & 22.2 \\
\hline Spring 2009 & 23.0 & 19.0 & 17.1 & 19.3 & 23.2 & 19.9 & 21.8 \\
\hline Fall 2011 & 24.6 & 25.1 & 24.3 & 21.6 & 22.6 & 25.1 & 24.1 \\
\hline Winter 2011 & 28.7 & 22.8 & 24.3 & 19.3 & 25.0 & 22.5 & 26.2 \\
\hline \multicolumn{8}{|l|}{ Altitude, $\mathrm{m}$, of residence } \\
\hline 1,976-2,321, quartile 1 & 25.4 & 26.5 & 23.9 & 23.8 & 24.7 & 26.7 & 25.1 \\
\hline $2,322-2,644$, quartile 2 & 25.1 & 23.2 & 24.3 & 23.8 & 25.6 & 26.7 & 24.9 \\
\hline $2,645-2,861$, quartile 3 & 24.6 & 19.9 & 25.7 & 29.3 & 24.7 & 25.7 & 25.0 \\
\hline $2,862-3,803$, quartile 4 & 24.9 & 30.3 & 26.1 & 23.1 & 25.0 & 20.9 & 24.9 \\
\hline
\end{tabular}

Overall, $36.7 \%$ of nasopharyngeal samples were from children who had received $\geq 2$ PCV7 doses and were considered vaccinated. Approximately $5.0 \%$ of samples were from children who had received aminopenicillins, cotrimoxazole, chloramphenicol, or furazolidone within the 7 days preceding sample collection.

Quantitative PCR detected S. pneumoniae in 68.9\% of nasopharyngeal samples; $78.9 \%$ of ARI and $65.3 \%$ of non-ARI samples were positive $(\mathrm{p}=0.06)$. Unadjusted logtransformed pneumococcal densities varied by ARI periods (online Technical Appendix).

Adjusted analyses showed that densities peaked during ARIs. In post hoc adjusted comparisons, densities were higher during the $1-7$ days pre-ARI $(p<0.0001)$, ARI $(p<0.0001)$, $1-7$ days post-ARI $(\mathrm{p}<0.0001)$, and $8-14$ days post-ARI $(\mathrm{p}=$ 0.007 ) than during the non-ARI period (Figure 1).

Of 450 ARI nasopharyngeal samples, 435 (97\%) had corresponding nasal swab samples available for identification of respiratory viruses; $299(68.7 \%)$ tested positive for at least 1 virus. Rhinovirus, which was detected in $44.6 \%$ (194/435) of samples, was the most common virus (online Technical Appendix). The median log-transformed pneumococcal densities of 299 virus-positive samples and 136 virus-negative samples were not significantly different (4.73 vs 3.94, respectively; adjusted $p=0.06$ ).

During ARI, the median log-transformed pneumococcal densities varied among virus groups: virus-negative (3.94, IQR $0.00-5.67 ; \mathrm{n}=136)$, nonrhinovirus (4.49, IQR 3.12-5.48; $\mathrm{n}=105)$, rhinovirus-only (4.91, IQR
$3.43-6.23 ; \mathrm{n}=147)$, and rhinovirus detected with other viruses samples (5.03, IQR 3.28-6.53; $\mathrm{n}=47$ ). In multivariable analyses, the only significant difference was between rhinovirus-only and virus-negative samples ( $\mathrm{p}=$ 0.02) (Figure 2).

For the colonization patterns assessment, 2,479 (69.3\%) nasopharyngeal samples had another sample collected $\leq 60$ days before the current sample; the median time between samples was 28 days. The median log-transformed pneumococcal densities among samples that represented new colonizations (5.14, IQR 3.56-6.24; $\mathrm{n}=411)$, serotype replacement $(5.49$, IQR 4.53-6.44; $\mathrm{n}=322)$, and serotype persistence $(5.79$, IQR 4.82-6.47; $\mathrm{n}=489)$ were compared. In multivariable analysis, serotype-replacement $(\mathrm{p}=0.005)$ and serotype-persistence $(\mathrm{p}=0.0003)$ samples had higher density than new colonization samples. The difference between serotype replacement and serotype persistence was not significant $(\mathrm{p}=0.2)$.

\section{Conclusions}

Our findings demonstrate a dynamic evolution of pneumococcal densities before, during, and after ARI episodes among young children. We observed a gradual increase in pneumococcal density leading up to an ARI episode, peak density during symptomatic ARI, and a decrease in density post-ARI to levels similar to those in baseline non-ARI periods.

Our observations of higher densities during ARI than non-ARI episodes align with those in studies from Vietnam 


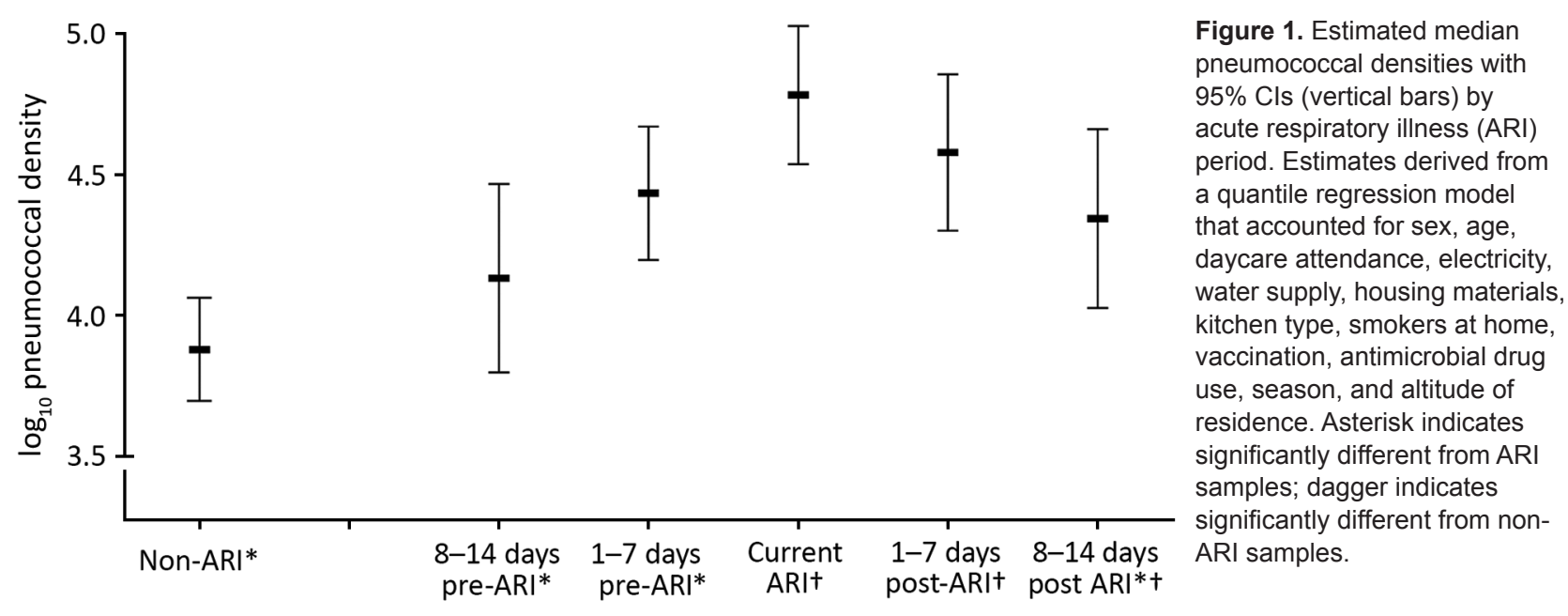

and South Africa $(14,15)$ and complement those assessments by illustrating the dynamic evolution of pneumococcal densities and the role of virus co-infections and pneumococcal colonization patterns. Unlike other studies that focused on hospitalized children, our community-based study showed relatively modest variations in nasopharyngeal pneumococcal density.

Rhinovirus detection was associated with increased pneumococcal density during ARI. Although we observed an even higher median pneumococcal density in samples co-infected with rhinovirus and other respiratory viruses, the number of observations was small and statistical power to demonstrate significant differences was limited.

Compared with new colonization in our study, serotype persistence and replacement were associated with higher pneumococcal density. Because many new colonizations might ultimately succumb to host mechanisms and fail to establish stable colonization (2), the observed lower densities might reflect a decline of pneumococcal populations as clearance evolved. Nevertheless, although statistically significant, the differences in density were relatively modest, and we cannot establish the precise time of colonization or clearance in our samples.

Our study has several limitations. ARI identification depended on the presence of cough or fever, which are subjective but widely used for routine ARI surveillance (5-7). Because our study used household-based rather than health facility-based surveillance, severe disease was infrequent, precluding detailed assessments of disease severity. Due to small numbers, we could not study serotype-specific pneumococcal densities. In addition, because the study was conducted in rural communities of Peru, caution is warranted when extrapolating our findings to other settings.

Our findings demonstrated that, among young children, nasopharyngeal pneumococcal density started increasing before the onset of ARI symptoms, peaked during symptomatic ARI, and decreased after symptoms subsided.

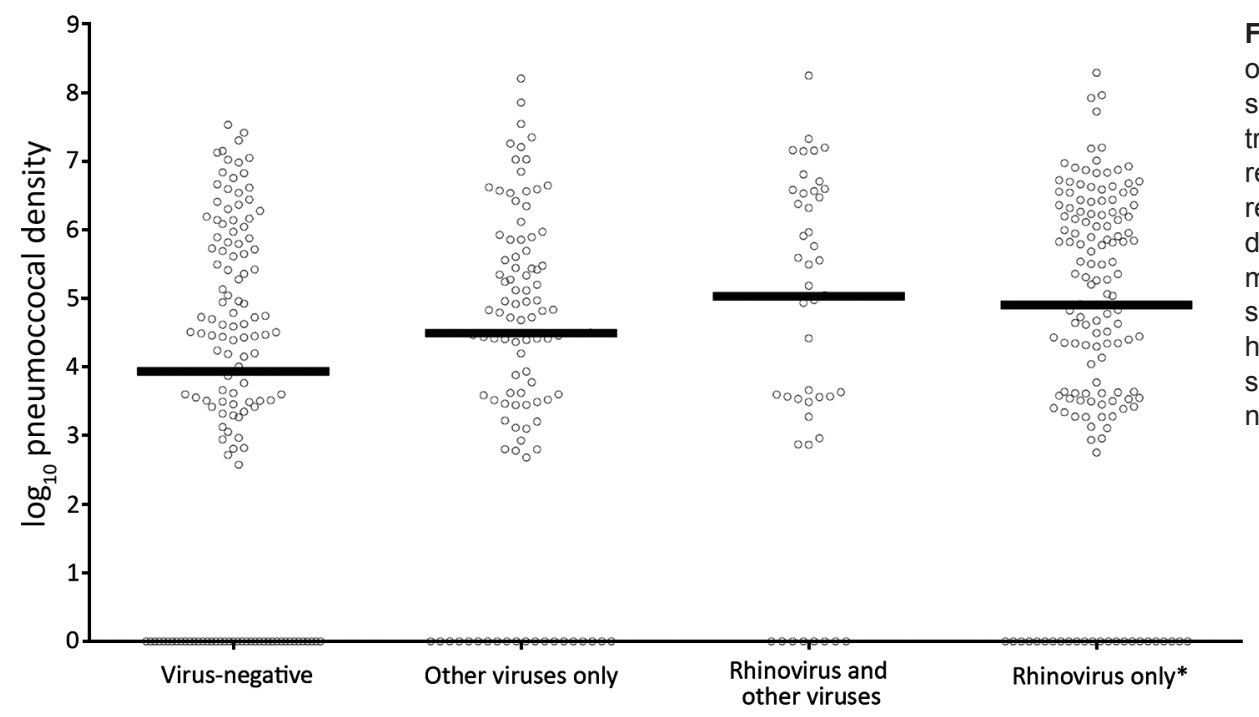

Figure 2. Pneumococcal densities of current acute respiratory illness samples subdivided by reverse transcription PCR detection of respiratory viruses. Each circle represents a single bacterial density measurement. The median for the samples of each subgroup is represented by a gray horizontal line. Asterisk indicates significantly different from virusnegative samples. 
Rhinovirus co-infection, serotype persistence, and serotype replacement were associated with increased nasopharyngeal pneumococcal density. Nasopharyngeal pneumococcal density is dynamic surrounding ARI episodes and likely driven by complex virus-bacteria-host interactions.

\section{Acknowledgments}

We are indebted to the communities of San Marcos, Cajamarca, Peru, for participating in this study and to the field workers and field supervisors whose efforts in difficult geographic areas and harsh weather conditions enabled the conduct of this study. We also acknowledge the approval and continuous support of the Cajamarca Health Region authorities.

This work was funded by a Vanderbilt University Clinical and Translational Science Award (grant UL1 RR024975) from the National Institutes of Health; an investigator-initiated research grant from Pfizer (IIR WS1898786 [0887X1-4492] to C.G.G. and IIR WS2079099 to J.E.V.); the Thrasher Research Fund (grant 02832-9 to C.G.G.); and a Thrasher Early Career Award (to L.M.H.).

C.G.G. has served as a consultant to Pfizer in unrelated work. M.R.G. receives grant funding from MedImmune. K.M.E. receives grant funding from Novartis in unrelated work. J.V.W. serves on a Scientific Advisory Board for Quidel and an Independent Data Monitoring Committee for GlaxoSmithKline, neither related to the present work. C.F.L. serves as a Scientific Advisor to Takeda and GlaxoSmithKline in subjects not related to the present work. All authors have submitted the International Committee of Medical Journal Editors Form for Disclosure of Potential Conflicts of Interest.

Mr. Fan is a medical student at Vanderbilt University School of Medicine in Nashville, Tennessee. His research interests include the epidemiology of respiratory illnesses and hostpathogen interactions.

\section{References}

1. Simell B, Auranen K, Käyhty H, Goldblatt D, Dagan R, O'Brien KL; Pneumococcal Carriage Group. The fundamental link between pneumococcal carriage and disease. Expert Rev Vaccines. 2012;11:841-55. http://dx.doi.org/10.1586/erv.12.53

2. Siegel SJ, Weiser JN. Mechanisms of bacterial colonization of the respiratory tract. Annu Rev Microbiol. 2015;69:425-44. http://dx.doi.org/10.1146/annurev-micro-091014-104209

3. Budge PJ, Griffin MR, Edwards KM, Williams JV, Verastegui H, Hartinger SM, et al.; RESPIRA-PERU Group. A household-based study of acute viral respiratory illnesses in Andean children. Pediatr Infect Dis J. 2014;33:443-7. http://dx.doi.org/10.1097/ INF.0000000000000135

4. Grijalva CG, Griffin MR, Edwards KM, Williams JV, Gil AI, Verastegui $\mathrm{H}$, et al. Cohort profile: the study of respiratory pathogens in Andean children. Int J Epidemiol. 2014;43:1021-30. http://dx.doi.org/10.1093/ije/dyt065

5. Lanata CF, Rudan I, Boschi-Pinto C, Tomaskovic L, Cherian T, Weber M, et al. Methodological and quality issues in epidemiological studies of acute lower respiratory infections in children in developing countries. Int J Epidemiol. 2004;33:1362-72. http://dx.doi.org/10.1093/ije/dyh229

6. Poehling KA, Edwards KM, Weinberg GA, Szilagyi P, Staat MA, Iwane MK, et al.; New Vaccine Surveillance Network. The underrecognized burden of influenza in young children. N Engl J Med. 2006;355:31-40. http://dx.doi.org/10.1056/NEJMoa054869

7. Gove $\mathrm{S}$; the WHO Working Group on Guidelines for Integrated Management of the Sick Child. Integrated management of childhood illness by outpatient health workers: technical basis and overview. Bull World Health Organ. 1997;75 (Suppl 1):7-24.

8. Griffin MR, Walker FJ, Iwane MK, Weinberg GA, Staat MA, Erdman DD; New Vaccine Surveillance Network Study Group. Epidemiology of respiratory infections in young children: insights from the new vaccine surveillance network. Pediatr Infect Dis J. 2004;23(Suppl):S188-92. http://dx.doi.org/10.1097/01. inf.0000144660.53024.64

9. Kodani M, Yang G, Conklin LM, Travis TC, Whitney CG, Anderson LJ, et al. Application of TaqMan low-density arrays for simultaneous detection of multiple respiratory pathogens. J Clin Microbiol. 2011;49:2175-82. http://dx.doi.org/10.1128/ JCM.02270-10

10. Klemenc J, Asad Ali S, Johnson M, Tollefson SJ, Talbot HK, Hartert TV, et al. Real-time reverse transcriptase PCR assay for improved detection of human metapneumovirus. J Clin Virol. 2012;54:371-5. http://dx.doi.org/10.1016/j.jcv.2012.05.005

11. Lu X, Holloway B, Dare RK, Kuypers J, Yagi S, Williams JV, et al. Real-time reverse transcription-PCR assay for comprehensive detection of human rhinoviruses. J Clin Microbiol. 2008;46:533-9. http://dx.doi.org/10.1128/JCM.01739-07

12. Hanke CR, Grijalva CG, Chochua S, Pletz MW, Hornberg C, Edwards KM, et al. Bacterial density, serotype distribution and antibiotic resistance of pneumococcal strains from the nasopharynx of Peruvian children before and after pneumococcal conjugate vaccine 7. Pediatr Infect Dis J. 2016;35:432-9. http://dx.doi.org/10.1097/INF.0000000000001030

13. World Health Organization. Revised WHO classification and treatment of pneumonia in children at health facilities: evidence summaries. Geneva: the Organization; 2014.

14. Vu HT, Yoshida LM, Suzuki M, Nguyen HA, Nguyen CD, Nguyen AT, et al. Association between nasopharyngeal load of Streptococcus pneumoniae, viral coinfection, and radiologically confirmed pneumonia in Vietnamese children. Pediatr Infect Dis J. 2011;30:11-8. http://dx.doi.org/10.1097/INF.0b013e3181f111a2

15. Wolter N, Tempia S, Cohen C, Madhi SA, Venter M, Moyes J, et al. High nasopharyngeal pneumococcal density, increased by viral coinfection, is associated with invasive pneumococcal pneumonia. J Infect Dis. 2014;210:1649-57. http://dx.doi.org/10.1093/ infdis/jiu326

Address for correspondence: Carlos G. Grijalva, Department of Health

Policy, Vanderbilt University School of Medicine, 2600 Village at

Vanderbilt, 1500 21st Ave, Nashville, TN, USA, 37212; email:

Carlos.grijalva@vanderbilt.edu 